\title{
ON MULTI-ORDER LOGARITHMIC POLYNOMIALS AND THEIR EXPLICIT FORMULAS, RECURRENCE RELATIONS, AND INEQUALITIES
}

\author{
FENG QI \\ Institute of Mathematics, Henan Polytechnic University, Jiaozuo City, Henan \\ Province, 454010, China; Department of Mathematics, College of Science, Tianjin \\ Polytechnic University, Tianjin City, 300387, China
}

\begin{abstract}
In the paper, the author introduces the notions "multi-order logarithmic numbers" and "multi-order logarithmic polynomials", establishes an explicit formula, an identity, and two recurrence relations by virtue of the Faà di Bruno formula and two identities of the Bell polynomials of the second kind in terms of the Stirling numbers of the first and second kinds, and constructs some determinantal inequalities, product inequalities, logarithmic convexity for multi-order logarithmic numbers and polynomials by virtue of some properties of completely monotonic functions.
\end{abstract}

\section{Multi-ORDer LOGARIthmic POLYNOMials}

Let $g(t)=e^{t}-1$ for $t \in \mathbb{R}$ and denote $\boldsymbol{x}_{m}=\left(x_{1}, x_{2}, \ldots, x_{m-1}, x_{m}\right)$ for $x_{k} \in \mathbb{R}$ and $1 \leq k \leq m$. Recently, the quantities $Q_{m, n}\left(\boldsymbol{x}_{m}\right)$ were defined by

$$
G\left(t ; \boldsymbol{x}_{m}\right)=\exp \left(x_{1} g\left(x_{2} g\left(\cdots x_{m-1} g\left(x_{m} g(t)\right) \cdots\right)\right)\right)=\sum_{n=0}^{\infty} Q_{m, n}\left(\boldsymbol{x}_{m}\right) \frac{t^{n}}{n !}
$$

and were called the Bell-Touchard polynomials [22. When $m=1$ and $x_{1}=1$, the quantities $Q_{1, n}(1)=B_{n}$ were called the Bell numbers [1, 5, 8, 17, 18, or exponential numbers [2] and were generalized and applied [1. When $m=1$ and $x_{1}=x$ is a variable, the quantities $Q_{1, n}(x)=B_{n}(x)=T_{n}(x)$ were called the Bell polynomials [20, 21, the Touchard polynomials [19, 22], or exponential polynomials [3, 4, 7] and were applied [9, 10, 11, 12, 19. In the paper [22, explicit formulas, recurrence relations, determinantal inequalities, product inequalities, logarithmic convexity, logarithmic concavity, and applications of $Q_{m, n}(x)$ were investigated. For more information on this topic, please refer to 22 and closely related references therein.

E-mail address: qifeng618@gmail.com, qifeng618@hotmail.com

2010 Mathematics Subject Classification. Primary 11B83; Secondary 11A25, 11B73, 11C08, 11C20, 15A15, 26A24, 26A48, 26C05, 26D05, 33B10, 34A05.

Key words and phrases. multi-order logarithmic number; multi-order logarithmic polynomial; explicit formula; identity; recurrence relation; inversion theorem; Bell polynomial of the second kind; Stirling number; determinantal inequality; product inequality; completely monotonic function; logarithmic convexity; Faà di Bruno formula.

This paper was typeset using $\mathcal{A M}_{\mathcal{M}} \mathcal{S}$-IATEX. 
According to the monograph [6, pp. 140-141], logarithmic polynomials $\mathrm{L}_{n}$ was defined by

$$
\ln \left(\sum_{n=0}^{\infty} g_{n} \frac{t^{n}}{n !}\right)=\sum_{n=1}^{\infty} \mathrm{L}_{n} \frac{t^{n}}{n !}
$$

where $g_{0}=G(a)=1, g_{n}=G^{(n)}(a)$ for $n \in \mathbb{N}$, and $G(x)$ is an infinitely differentiable function at $x=a$. In other words, the logarithmic polynomials $\mathrm{L}_{n}$ are expressions for the $n$th derivative of $\ln G(x)$ at the point $x=a$. See also [23, Section 5.2].

We now introduce two new notions "multi-order logarithmic numbers" and "multiorder logarithmic polynomials".

Definition 1.1. For $x_{k} \in \mathbb{R}$ and $1 \leq k \leq m$, denote $\boldsymbol{x}_{m}=\left(x_{1}, x_{2}, \ldots, x_{m-1}, x_{m}\right)$. Let $h(t)=\ln (1+t)$ for $t>-1$. Define $H\left(t ; \boldsymbol{x}_{m}\right)$ and $L_{m, n}\left(\boldsymbol{x}_{m}\right)$ by

$$
\begin{gathered}
H\left(t ; \boldsymbol{x}_{m}\right)=\ln \left(1+x_{1} \ln \left(1+x_{2} \ln \left(1+\cdots+x_{m-1} \ln \left(1+x_{m} \ln (1+t)\right) \cdots\right)\right)\right) \\
=h\left(x_{1} h\left(x_{2} h\left(\cdots x_{m-1} h\left(x_{m} h(t)\right) \cdots\right)\right)\right)=\sum_{n=0}^{\infty} L_{m, n}\left(\boldsymbol{x}_{m}\right) \frac{t^{n}}{n !} .
\end{gathered}
$$

We call $L_{m, n}\left(\boldsymbol{x}_{m}\right)$ higher order logarithmic polynomials, logarithmic polynomials of order $m, m$-variate logarithmic polynomials, multivariate logarithmic polynomials, logarithmic polynomials of $m$ variables $x_{1}, x_{2}, \ldots, x_{m}$, multi-order logarithmic polynomials alternatively. When $x_{1}=x_{2}=\cdots=x_{m-1}=x_{m}=1$, we denote $L_{m, n}(1, \ldots, 1)$ by $L_{m, n}$ and call them higher order logarithmic numbers, logarithmic numbers of order $m$, and multi-order logarithmic numbers alternatively.

It is not difficult to see that each of the generating functions $G\left(t ; \boldsymbol{x}_{m}\right)$ and $H\left(t ; \boldsymbol{x}_{m}\right)$ is an inverse function of another one.

Since the power series

$$
\ln (1+t)=\sum_{n=1}^{\infty}(-1)^{n-1} \frac{t^{n}}{n}=\sum_{n=1}^{\infty}(-1)^{n-1}(n-1) ! \frac{t^{n}}{n !},
$$

we write $L_{0,0}=0$ and $L_{0, n}=(-1)^{n-1}(n-1)$ ! for $n \geq 1$.

From the limit $\lim _{t \rightarrow 0} H\left(t ; \boldsymbol{x}_{m}\right)=0$, it follows that $L_{m, 0}\left(\boldsymbol{x}_{m}\right)=0$ for $m \geq 1$.

By the software MATHEMATiCA, we can obtain

$$
\ln [1+\ln (1+t)]=t-t^{2}+\frac{7 t^{3}}{6}-\frac{35 t^{4}}{24}+\frac{19 t^{5}}{10}-\frac{917 t^{6}}{360}+\frac{8791 t^{7}}{2520}-\cdots
$$

and

$$
\begin{gathered}
\ln [1+x \ln (1+t)]=x t-\frac{1}{2} x(1+x) t^{2}+\frac{1}{6} x\left(2 x^{2}+3 x+2\right) t^{3} \\
-\frac{1}{24} x\left(6 x^{3}+12 x^{2}+11 x+6\right) t^{4}+\frac{1}{60} x\left(12 x^{4}+30 x^{3}+35 x^{2}+25 x+12\right) t^{5} \\
-\frac{1}{360} x\left(60 x^{5}+180 x^{4}+255 x^{3}+225 x^{2}+137 x+60\right) t^{6}+\cdots
\end{gathered}
$$

which imply that the first few 1 -order logarithmic numbers $L_{1, n}$ for $1 \leq n \leq 7$ are

$$
1, \quad-2, \quad 7, \quad-35, \quad 228, \quad-1834, \quad 17582
$$

and that the first few 1-order logarithmic polynomials $L_{1, n}(x)$ for $1 \leq n \leq 6$ are

$$
\begin{gathered}
x, \quad-x(1+x), \quad x\left(2+3 x+2 x^{2}\right), \quad-x\left(6+11 x+12 x^{2}+6 x^{3}\right), \\
x\left(12+25 x+35 x^{2}+30 x^{3}+12 x^{4}\right),
\end{gathered}
$$




$$
-x\left(60+137 x+225 x^{2}+255 x^{3}+180 x^{4}+60 x^{5}\right) .
$$

Basing on the above concrete examples, we guess that all multi-order logarithmic numbers $(-1)^{n-1} L_{m, n}$ are positive integers and that all multi-order logarithmic polynomials $(-1)^{n-1} L_{m, n}\left(\boldsymbol{x}_{m}\right)$ are positive integer polynomials of variables $x_{1}, x_{2}, \ldots, x_{m}$ with degree $m \times n$ for $m, n \in \mathbb{N}$. This guess will be verified in next section.

\section{RECURRENCE RELATIONS AND EXPLiCIT FORMULAS}

In this section, we present two recurrence relations, one explicit formula, and one identity for multi-order logarithmic polynomials $L_{m, n}\left(\boldsymbol{x}_{m}\right)$.

Theorem 2.1. For $m, n \in \mathbb{N}$, multi-order logarithmic polynomials $L_{m, n}\left(\boldsymbol{x}_{m}\right)$ can be computed by the forward recurrence relation

$$
L_{m, n}\left(\boldsymbol{x}_{m}\right)=\sum_{k=0}^{n} s(n, k) L_{m-1, k}\left(\boldsymbol{x}_{m-1}\right) x_{m}^{k}
$$

by the backward recurrence relation

$$
L_{m-1, n}\left(\boldsymbol{x}_{m-1}\right) x_{m}^{n}=\sum_{k=0}^{n} S(n, k) L_{m, k}\left(\boldsymbol{x}_{m}\right),
$$

by the explicit formula

$$
L_{m, n}\left(\boldsymbol{x}_{m}\right)=\left[\prod_{q=1}^{m} \sum_{\ell_{q}=1}^{\ell_{q-1}} s\left(\ell_{q-1}, \ell_{q}\right) x_{m-q+1}^{\ell_{q}}\right] s\left(\ell_{m}, 1\right),
$$

and by the identity

$$
\left[\prod_{q=1}^{m} \frac{1}{x_{q}^{\ell_{q-1}}} \sum_{\ell_{q}=1}^{\ell_{q-1}} S\left(\ell_{q-1}, \ell_{q}\right)\right] L_{m, \ell_{m}}\left(\boldsymbol{x}_{m}\right)=s(n, 1)
$$

for $\ell_{0}=n$, where $s(n, k)$ and $S(n, k)$ for $n \geq k \geq 0$ respectively stand for the Stirling numbers of the first and second kinds which can be generated respectively by

$$
\frac{[\ln (1+x)]^{k}}{k !}=\sum_{n=k}^{\infty} s(n, k) \frac{x^{n}}{n !}, \quad|x|<1
$$

and

$$
\frac{\left(e^{x}-1\right)^{k}}{k !}=\sum_{n=k}^{\infty} S(n, k) \frac{x^{n}}{n !} .
$$

Proof. In combinatorics, the Bell polynomials of the second kind $\mathrm{B}_{n, k}\left(\boldsymbol{x}_{n-k+1}\right)$ are defined by

$$
\mathrm{B}_{n, k}\left(\boldsymbol{x}_{n-k+1}\right)=\sum_{\substack{\ell_{1}, \ldots, \ell_{n} \in\{0\} \cup \mathbb{N} \\ \sum_{i=1}^{n} i \ell_{i}=n \\ \sum_{i=1}^{n} \ell_{i}=k}} \frac{n !}{\prod_{i=1}^{n-k+1} \ell_{i} !} \prod_{i=1}^{n-k+1}\left(\frac{x_{i}}{i !}\right)^{\ell_{i}}
$$

for $n \geq k \geq 0$, see [6, p. 134, Theorem A], and satisfy two identities

$$
\mathrm{B}_{n, k}\left(a b x_{1}, a b^{2} x_{2}, \ldots, a b^{n-k+1} x_{n-k+1}\right)=a^{k} b^{n} \mathrm{~B}_{n, k}\left(x_{1}, x_{2}, \ldots, x_{n-k+1}\right)
$$


and

$$
\mathrm{B}_{n, k}(0 !, 1 !, 2 !, \ldots,(n-k) !)=(-1)^{n-k} s(n, k),
$$

see [6, p. 135, Theorem B], where $a$ and $b$ are any complex numbers. The Faà di Bruno formula for higher order derivatives of composite functions can be described in terms of the Bell polynomials of the second kind $\mathrm{B}_{n, k}\left(\boldsymbol{x}_{n-k+1}\right)$ by

$$
\frac{\mathrm{d}^{n}}{\mathrm{~d} t^{n}} f \circ q(x)=\sum_{k=0}^{n} f^{(k)}(q(x)) \mathrm{B}_{n, k}\left(q^{\prime}(x), q^{\prime \prime}(x), \ldots, q^{(n-k+1)}(x)\right),
$$

see [6, p. 139, Theorem C].

In [24, p. 171, Theorem 12.1], it is stated that, if $b_{\alpha}$ and $a_{k}$ are a collection of constants independent of $n$, then

$$
a_{n}=\sum_{\alpha=0}^{n} S(n, \alpha) b_{\alpha} \quad \text { if and only if } \quad b_{n}=\sum_{k=0}^{n} s(n, k) a_{k} .
$$

By Definition 1.1 and the formulas 2.7), 2.5), and 2.6 in sequence, we have

$$
\begin{gathered}
L_{1, n}(x)=\lim _{t \rightarrow 0} \frac{\partial^{n} H(t ; x)}{\partial t^{n}}=\lim _{t \rightarrow 0} \frac{\partial^{n} \ln [1+x \ln (1+t)]}{\partial t^{n}} \\
=\lim _{t \rightarrow 0} \sum_{k=0}^{n} \frac{\partial^{k} \ln (1+v)}{\partial v^{k}} \mathrm{~B}_{n, k}\left(\frac{x}{1+t}, \frac{-x}{(1+t)^{2}}, \ldots, \frac{(-1)^{n-k} x(n-k) !}{(1+t)^{n-k+1}}\right) \\
=\lim _{t \rightarrow 0} \sum_{k=1}^{n} \frac{(-1)^{k-1}(k-1) !}{(1+v)^{k}}\left(\frac{1}{1+t}\right)^{n} x^{k}(-1)^{k+n} \mathrm{~B}_{n, k}(0 !, 1 !, \ldots,(n-k) !) \\
=\sum_{k=1}^{n}(-1)^{k-1}(k-1) ! x^{k} s(n, k)=\sum_{k=1}^{n}(-1)^{k-1} s(n, k)(k-1) ! x^{k},
\end{gathered}
$$

where $v=v(t)=x \ln (1+t)$.

For $m \geq 2$, the generating function $H\left(t ; \boldsymbol{x}_{m}\right)$ can be rewritten as

$$
H\left(t ; \boldsymbol{x}_{m}\right)=H\left(x_{m} h(t) ; \boldsymbol{x}_{m-1}\right) .
$$

Hence, making use of the formulas (2.7), 2.5), and 2.6 in sequence gives

$$
\begin{aligned}
\frac{\partial^{n} H\left(t ; \boldsymbol{x}_{m}\right)}{\partial t^{n}} & =\sum_{k=0}^{n} \frac{\partial^{k} H\left(u ; \boldsymbol{x}_{m-1}\right)}{\partial u^{k}} \mathrm{~B}_{n, k}\left(x_{m} h^{\prime}(t), \ldots, x_{m} h^{(n-k+1)}(t)\right) \\
& =\sum_{k=0}^{n} \frac{\partial^{k} H\left(u ; \boldsymbol{x}_{m-1}\right)}{\partial u^{k}} x_{m}^{k} \mathrm{~B}_{n, k}\left(\frac{1}{1+t}, \ldots, \frac{(-1)^{n-k}(n-k) !}{(1+t)^{n-k+1}}\right) \\
& =\sum_{k=0}^{n} \frac{\partial^{k} H\left(u ; \boldsymbol{x}_{m-1}\right)}{\partial u^{k}} x_{m}^{k}\left(\frac{1}{1+t}\right)^{n}(-1)^{n+k} \mathrm{~B}_{n, k}(0 !, 1 !, \ldots,(n-k) !) \\
& =\sum_{k=0}^{n} \frac{\partial^{k} H\left(u ; \boldsymbol{x}_{m-1}\right)}{\partial u^{k}} x_{m}^{k}\left(\frac{1}{1+t}\right)^{n} s(n, k),
\end{aligned}
$$

where $u=u(t)=x_{m} h(t)=x_{m} \ln (1+t)$. Further letting $t \rightarrow 0$ and considering the definition in 1.1 yield the recurrence relation 2.1.

Computing the recurrence relation 2.1 results in

$$
L_{m, n}\left(\boldsymbol{x}_{m}\right)=\sum_{\ell_{1}=0}^{n} s\left(n, \ell_{1}\right) \sum_{\ell_{2}=0}^{\ell_{1}} s\left(\ell_{1}, \ell_{2}\right) L_{m-2, \ell_{2}}\left(\boldsymbol{x}_{m-2}\right) x_{m-1}^{\ell_{2}} x_{m}^{\ell_{1}}=\cdots
$$




$$
\begin{aligned}
=\sum_{\ell_{1}=0}^{n} s\left(n, \ell_{1}\right) & \sum_{\ell_{2}=0}^{\ell_{1}} s\left(\ell_{1}, \ell_{2}\right) \cdots \sum_{\ell_{m-1}=0}^{\ell_{m}-2} s\left(\ell_{m-2}, \ell_{m-1}\right) L_{1, \ell_{m-1}}\left(x_{1}\right) x_{2}^{\ell_{m-1}} \cdots x_{m-1}^{\ell_{2}} x_{m}^{\ell_{1}} \\
= & \sum_{\ell_{1}=0}^{n} s\left(n, \ell_{1}\right) \sum_{\ell_{2}=0}^{\ell_{1}} s\left(\ell_{1}, \ell_{2}\right) \cdots \sum_{\ell_{m-1}=0}^{\ell_{m-2}} s\left(\ell_{m-2}, \ell_{m-1}\right) \sum_{\ell_{m}=1}^{\ell_{m-1}} \\
\times & (-1)^{\ell_{m}-1} s\left(\ell_{m-1}, \ell_{m}\right)\left(\ell_{m}-1\right) ! x_{1}^{\ell_{m}} x_{2}^{\ell_{m-1}} \cdots x_{m-1}^{\ell_{2}} x_{m}^{\ell_{1}} \\
= & {\left[\prod_{q=1}^{m} \sum_{\ell_{q}=1}^{\ell_{q-1}} s\left(\ell_{q-1}, \ell_{q}\right) x_{m-q+1}^{\ell_{q}}\right](-1)^{\ell_{m}-1}\left(\ell_{m}-1\right) ! . }
\end{aligned}
$$

The explicit formula (2.3) thus follows.

Applying the above mentioned inversion theorem, [24, Theorem 12.1], to the recurrence relation 2.1 arrives at the backward recurrence relation 2.2 readily.

Applying the above mentioned inversion theorem inductively and recursively to the backward recurrence relation $(2.2)$ produces

$$
\begin{gathered}
\sum_{k=1}^{n}(-1)^{k-1} s(n, k)(k-1) ! x_{1}^{k} x_{2}^{n}=L_{1, n}\left(x_{1}\right) x_{2}^{n}=\sum_{k=0}^{n} S(n, k) L_{2, k}\left(\boldsymbol{x}_{2}\right) \\
=\sum_{\ell_{1}=0}^{n} S\left(n, \ell_{1}\right) \frac{1}{x_{3}^{\ell_{1}}} \sum_{k=0}^{\ell_{1}} S\left(\ell_{1}, k\right) L_{3, k}\left(\boldsymbol{x}_{3}\right)=\cdots \\
=\sum_{\ell_{1}=0}^{n} S\left(n, \ell_{1}\right) \frac{1}{x_{3}^{\ell_{1}}} \sum_{\ell_{2}=0}^{\ell_{1}} S\left(\ell_{1}, \ell_{2}\right) \cdots \frac{1}{x_{m}^{\ell_{m}-2}} \sum_{\ell_{m-1}=0}^{\ell_{m-2}} S\left(\ell_{m-2}, \ell_{m-1}\right) L_{m, \ell_{m-1}}\left(\boldsymbol{x}_{m}\right) .
\end{gathered}
$$

Consequently,

$$
\begin{aligned}
(-1)^{n-1}(n-1) ! x_{1}^{n}=\sum_{k=1}^{n} S(n, k) \frac{1}{x_{2}^{k}} \sum_{\ell_{1}=0}^{k} S\left(k, \ell_{1}\right) \frac{1}{x_{3}^{\ell_{1}}} \sum_{\ell_{2}=0}^{\ell_{1}} S\left(\ell_{1}, \ell_{2}\right) \cdots \\
\\
\frac{1}{x_{m}^{\ell_{m-2}}} \sum_{\ell_{m-1}=0}^{\ell_{m-2}} S\left(\ell_{m-2}, \ell_{m-1}\right) L_{m, \ell_{m-1}}\left(\boldsymbol{x}_{m}\right)
\end{aligned}
$$

which can be rearranged as (2.4). This can also be derived from applying the inversion theorem in 2.8 to the explicit formula 2.3 . The proof of Theorem 2.1 is complete.

Remark 2.1. When letting $m=1,2,3$, the explicit formula 2.3 and the identity (2.4) reduce to

$$
\begin{gathered}
L_{1, n}(x)=\sum_{\ell=1}^{n} s(n, \ell) s(\ell, 1) x^{\ell}, \quad L_{2, n}(x, y)=\sum_{\ell=1}^{n} s(n, \ell) y^{\ell} \sum_{k=1}^{\ell} s(\ell, k) s(k, 1) x^{k} \\
L_{3, n}(x, y, z)=\sum_{i=1}^{n} s(n, i) z^{i} \sum_{j=1}^{i} s(i, j) y^{j} \sum_{k=1}^{j} s(j, k) s(k, 1) x^{k}
\end{gathered}
$$

and

$$
\sum_{\ell=1}^{n} S(n, \ell) L_{1, \ell}(x)=s(n, 1) x^{n}, \quad \sum_{\ell=1}^{n} \frac{S(n, \ell)}{y^{\ell}} \sum_{k=1}^{\ell} S(\ell, k) L_{2, k}(x, y)=s(n, 1) x^{n},
$$




$$
\sum_{i=1}^{n} \frac{S(n, i)}{y^{i}} \sum_{j=1}^{i} \frac{S(i, j)}{z^{j}} \sum_{k=1}^{j} S(j, k) L_{3, k}(x, y, z)=s(n, 1) x^{n}
$$

respectively.

Remark 2.2. When taking $x_{1}=x_{2}=\cdots=x_{m}=1$ in Theorem 2.1, we can derive two recurrence relations, one explicit formula, and one identity for multi-order logarithmic numbers $L_{m, n}$.

\section{INEQUALITIES}

In this section, we will construct some determinantal and product inequalities for multi-order logarithmic polynomials $L_{m, n}\left(\boldsymbol{x}_{m}\right)$ and derive the logarithmic convexity and logarithmic concavity for the sequences $\left\{L_{m, n}(x)\right\}_{n \geq 1}$ and $\left\{\frac{L_{m, n}(x)}{n !}\right\}_{n \geq 1}$ respectively.

Theorem 3.1. Let $q \geq 1$ be a positive integer, let $\left|e_{i j}\right|_{q}$ denote a determinant of order $q$ with elements $e_{i j}$, and let $x_{k}>0$ for $1 \leq k \leq m$.

(1) If $a_{i}$ for $1 \leq i \leq q$ are non-negative integers, then

$$
\left|L_{m, a_{i}+a_{j}+1}\left(\boldsymbol{x}_{m}\right)\right|_{q} \geq 0 \quad \text { and } \quad\left|(-1)^{a_{i}+a_{j}} L_{m, a_{i}+a_{j}+1}\left(\boldsymbol{x}_{m}\right)\right|_{q} \geq 0 .
$$

(2) If $a=\left(a_{1}, a_{2}, \ldots, a_{q}\right)$ and $b=\left(b_{1}, b_{2}, \ldots, b_{q}\right)$ are non-increasing $q$-tuples of non-negative integers such that $\sum_{i=1}^{k} a_{i} \geq \sum_{i=1}^{k} b_{i}$ for $1 \leq k \leq q-1$ and $\sum_{i=1}^{q} a_{i}=\sum_{i=1}^{q} b_{i} \triangleq P$, then

$$
(-1)^{P} \prod_{i=1}^{q} L_{m, a_{i}+1}\left(\boldsymbol{x}_{m}\right) \geq(-1)^{P} \prod_{i=1}^{q} L_{m, b_{i}+1}\left(\boldsymbol{x}_{m}\right) .
$$

Proof. Recall from [15, Chapter XIII], 25, Chapter 1], and [28, Chapter IV] that an infinitely differentiable function $f$ is said to be completely monotonic on an interval $I$ if it satisfies $(-1)^{k} f^{(k)}(x) \geq 0$ on $I$ for all $k \geq 0$. Recall also from [25. p. 21, Definition 3.1] that a nonnegative function $f:(0, \infty) \rightarrow \mathbb{R}$ is a Bernstein function if its first derivative $f^{\prime}$ is completely monotonic on $(0, \infty)$. Item (iii) in [25, p. 28, Corollary 3.8] reads that the composition of two Bernstein functions is still a Bernstein function. Therefore, since the function $h(t)=\ln (1+t)$ is a Bernstein function, when $x_{k}>0$ for $1 \leq k \leq m$, the generating function $H\left(t ; \boldsymbol{x}_{m}\right)$ is a Bernstein function and its first derivative $H\left(t ; \boldsymbol{x}_{m}\right)$ is a completely monotonic function on $[0, \infty)$. By Definition 1.1, we have

$$
L_{m, n}\left(\boldsymbol{x}_{m}\right)=\lim _{t \rightarrow 0} \frac{\partial^{n} H\left(t ; \boldsymbol{x}_{m}\right)}{\partial t^{n}}=\lim _{t \rightarrow 0} \frac{\partial^{n-1} H_{t}^{\prime}\left(t ; \boldsymbol{x}_{m}\right)}{\partial t^{n-1}}, \quad m, n \in \mathbb{N} .
$$

In [14] and [15, p. 367], it was proved that if $f(t)$ is completely monotonic on $[0, \infty)$, then

$$
\left|f^{\left(a_{i}+a_{j}\right)}(t)\right|_{q} \geq 0 \quad \text { and } \quad\left|(-1)^{a_{i}+a_{j}} f^{\left(a_{i}+a_{j}\right)}(t)\right|_{q} \geq 0
$$

Applying $f(t)$ to the first derivative $H_{t}^{\prime}\left(t ; \boldsymbol{x}_{m}\right)$ in 3.4 , taking the limit $t \rightarrow 0^{+}$, and considering the equation 3.3 give

$$
\lim _{t \rightarrow 0^{+}}\left|\left[H_{t}^{\prime}\left(t ; \boldsymbol{x}_{m}\right)\right]_{t}^{\left(a_{i}+a_{j}\right)}\right|_{q}=\left|L_{m, a_{i}+a_{j}+1}\left(\boldsymbol{x}_{m}\right)\right|_{q} \geq 0
$$


and

$$
\lim _{t \rightarrow 0^{+}}\left|(-1)^{a_{i}+a_{j}}\left[H_{t}^{\prime}\left(t ; \boldsymbol{x}_{m}\right)\right]_{t}^{\left(a_{i}+a_{j}\right)}\right|_{q}=\left|(-1)^{a_{i}+a_{j}} L_{m, a_{i}+a_{j}+1}\left(\boldsymbol{x}_{m}\right)\right|_{q} \geq 0
$$

The determinantal inequalities in (3.1) follow immediately.

In [15, p. 367, Theorem 2], it was stated that if $f(t)$ is a completely monotonic function on $[0, \infty)$, then

$$
\prod_{i=1}^{q}\left[(-1)^{a_{i}} f^{\left(a_{i}\right)}(t)\right] \geq \prod_{i=1}^{q}\left[(-1)^{b_{i}} f^{\left(b_{i}\right)}(t)\right] .
$$

Applying $f(t)$ to the derivative $H_{t}^{\prime}\left(t ; \boldsymbol{x}_{m}\right)$ in $(3.5)$, taking the limit $t \rightarrow 0^{+}$, and considering the equation 3.3 result in

$$
\begin{aligned}
\lim _{t \rightarrow 0^{+}} \prod_{i=1}^{q}\left[(-1)^{a_{i}}\right. & \left.\left(H_{t}^{\prime}\left(t ; \boldsymbol{x}_{m}\right)\right)_{t}^{\left(a_{i}\right)}\right]=\prod_{i=1}^{q}\left[(-1)^{a_{i}} L_{m, a_{i}+1}\left(\boldsymbol{x}_{m}\right)\right] \\
& \geq \lim _{t \rightarrow 0^{+}} \prod_{i=1}^{q}\left[(-1)^{b_{i}}\left(H_{t}^{\prime}\left(t ; \boldsymbol{x}_{m}\right)\right)_{t}^{\left(b_{i}\right)}\right]=\prod_{i=1}^{q}\left[(-1)^{b_{i}} L_{m, b_{i}+1}\left(\boldsymbol{x}_{m}\right)\right] .
\end{aligned}
$$

The product inequality 3.2 thus follows. Theorem 3.1 is proved.

Corollary 3.1. Let $x_{k}>0$ for $1 \leq k \leq m$. If $\ell \geq 0$ and $q \geq k \geq 0$, then

$$
(-1)^{(k+\ell) q}\left[L_{m, q+\ell+1}\left(\boldsymbol{x}_{m}\right)\right]^{k}\left[L_{m, \ell+1}\left(\boldsymbol{x}_{m}\right)\right]^{q-k} \geq(-1)^{(k+\ell) q}\left[L_{m, k+\ell+1}\left(\boldsymbol{x}_{m}\right)\right]^{q} .
$$

Proof. This follows from taking

$$
a=(\overbrace{q+\ell, \ldots, q+\ell}^{k}, \overbrace{\ell, \ldots, \ell}^{q-k}) \text { and } b=(k+\ell, k+\ell, \ldots, k+\ell)
$$

in the inequality 3.2 . The proof of Corollary 3.1 is complete.

Theorem 3.2. Let $x_{k}>0$ for $1 \leq k \leq m$. Then $\left\{(-1)^{n-1} L_{m, n}\left(\boldsymbol{x}_{m}\right)\right\}_{n \geq 1}$ is a logarithmically convex sequence.

Proof. In [15, p. 369] and [16, p. 429, Remark], it was obtained that if $f(t)$ is a completely monotonic function such that $f^{(k)}(t) \neq 0$ for $k \geq 0$, then the sequence

$$
\ln \left[(-1)^{k-1} f^{(k-1)}(t)\right], \quad k \geq 1
$$

is convex. Applying this result to $H^{\prime}\left(t ; \boldsymbol{x}_{m}\right)$ figures out that the sequence

$$
\ln \left[(-1)^{k-1}\left(H^{\prime}\left(t ; \boldsymbol{x}_{m}\right)\right)_{t}^{(k-1)}\right] \rightarrow \ln \left[(-1)^{k-1} L_{m, k}\left(\boldsymbol{x}_{m}\right)\right], \quad t \rightarrow 0^{+}
$$

for $k \geq 1$ is convex. Equivalently speaking, the sequence $\left\{(-1)^{n-1} L_{m, n}\left(\boldsymbol{x}_{m}\right)\right\}_{n \geq 1}$ is logarithmically convex.

Letting $\ell \geq 1, n=2, a_{1}=\ell+2, a_{2}=\ell$, and $b_{1}=b_{2}=\ell+1$ in the inequality 3.2 alternatively leads to

$$
L_{m, \ell+1}\left(\boldsymbol{x}_{m}\right) L_{m, \ell+3}\left(\boldsymbol{x}_{m}\right) \geq L_{m, \ell+2}^{2}\left(\boldsymbol{x}_{m}\right)
$$

which implies that the positive sequence $\left\{(-1)^{n-1} L_{m, n}\left(\boldsymbol{x}_{m}\right)\right\}_{n \geq 2}$ is logarithmically convex. The proof of Theorem 3.2 is complete. 
Theorem 3.3. Let $x_{k}>0$ for $1 \leq k \leq m$. For $q \geq 0$ and $n \in \mathbb{N}$, we have

$$
\left[(-1)^{(n+1)(q+1)} \prod_{\ell=0}^{n} L_{m, q+2 \ell+2}\left(\boldsymbol{x}_{m}\right)\right]^{1 /(n+1)} \geq\left[(-1)^{n q} \prod_{\ell=0}^{n-1} L_{m, q+2 \ell+3}\left(\boldsymbol{x}_{m}\right)\right]^{1 / n} \text {. }
$$

Proof. If $f(t)$ is a completely monotonic function on $(0, \infty)$, then, by the convexity of the sequence (3.6) and Nanson's inequality listed in [13, p. 205, 3.2.27],

$$
\left[\prod_{\ell=0}^{n}(-1)^{q+2 \ell+1} f^{(q+2 \ell+1)}(t)\right]^{1 /(n+1)} \geq\left[\prod_{\ell=1}^{n}(-1)^{q+2 \ell} f^{(q+2 \ell)}(t)\right]^{1 / n}
$$

for $q \geq 0$. Replacing $f(t)$ by $H^{\prime}\left(t ; \boldsymbol{x}_{m}\right)$ in the above inequality results in

$$
\left[\prod_{\ell=0}^{n}(-1)^{q+1}\left(H^{\prime}\left(t ; \boldsymbol{x}_{m}\right)\right)_{t}^{(q+2 \ell+1)}\right]^{1 /(n+1)} \geq\left[\prod_{\ell=1}^{n}(-1)^{q}\left(H^{\prime}\left(t ; \boldsymbol{x}_{m}\right)\right)_{t}^{(q+2 \ell)}\right]^{1 / n}
$$

for $q \geq 0$. Letting $t \rightarrow 0^{+}$in the above inequality leads to (3.7). The proof of Theorem 3.3 is complete.

Theorem 3.4. Let $x_{k}>0$ for $1 \leq k \leq m$. If $\ell \geq 0, n \geq k \geq q, 2 k \geq n$, and $2 q \geq n$, then

$$
(-1)^{n} L_{m, k+\ell+1}\left(\boldsymbol{x}_{m}\right) L_{m, n-k+\ell+1}\left(\boldsymbol{x}_{m}\right) \geq(-1)^{n} L_{m, q+\ell+1}\left(\boldsymbol{x}_{m}\right) L_{m, n-q+\ell+1}\left(\boldsymbol{x}_{m}\right)
$$

and

$$
(-1)^{n k}\left[L_{m, k+1}\left(\boldsymbol{x}_{m}\right)\right]^{n} \leq(-1)^{n k}\left[L_{m, n+1}\left(\boldsymbol{x}_{m}\right)\right]^{k}\left[L_{m, 1}\left(\boldsymbol{x}_{m}\right)\right]^{n-k} .
$$

Proof. In [26, p. 397, Theorem D], it was recovered that, if $f(t)$ is a completely monotonic function on $(0, \infty)$ and if $n \geq k \geq q, k \geq n-k$, and $q \geq n-q$, then

$$
(-1)^{n} f^{(k)}(t) f^{(n-k)}(t) \geq(-1)^{n} f^{(q)}(t) f^{(n-q)}(t) .
$$

Replacing $f(t)$ by the function $(-1)^{\ell}\left[H^{\prime}\left(t ; \boldsymbol{x}_{m}\right)\right]_{t}^{(\ell)}$ in the above inequality leads to

$$
\begin{aligned}
& (-1)^{n}\left[H^{\prime}\left(t ; \boldsymbol{x}_{m}\right)\right]_{t}^{(k+\ell)}\left[H^{\prime}\left(t ; \boldsymbol{x}_{m}\right)\right]_{t}^{(n-k+\ell)} \\
& \quad \geq(-1)^{n}\left[H^{\prime}\left(t ; \boldsymbol{x}_{m}\right)\right]_{t}^{(q+\ell)}\left[H^{\prime}\left(t ; \boldsymbol{x}_{m}\right)\right]_{t}^{(n-q+\ell)} .
\end{aligned}
$$

Further taking $t \rightarrow 0^{+}$finds the inequality (3.8).

When $f(t)$ is a completely monotonic function, the inequality

$$
(-1)^{n k}\left[f^{(k)}(t)\right]^{n} \leq(-1)^{n k}\left[f^{(n)}(t)\right]^{k} f^{n-k}(t)
$$

is valid for $0<k<n$. See [15, p. 368]. Substituting $H^{\prime}\left(t ; \boldsymbol{x}_{m}\right)$ for $f(t)$ reveals

$$
(-1)^{n k}\left[\left(H^{\prime}\left(t ; \boldsymbol{x}_{m}\right)\right)^{(k)}\right]^{n} \leq(-1)^{n k}\left[\left(H^{\prime}\left(t ; \boldsymbol{x}_{m}\right)\right)^{(n)}\right]^{k}\left[H^{\prime}\left(t ; \boldsymbol{x}_{m}\right)\right]^{n-k} \text {. }
$$

Letting $t \rightarrow 0^{+}$leads to 3.9 . The proof of Theorem 3.4 is complete.

Theorem 3.5. Let $x_{k}>0$ for $1 \leq k \leq m$. For $\ell \geq 0$ and $q, n \in \mathbb{N}$, let

$$
\begin{aligned}
\mathcal{G}_{m, \ell, q, n}= & L_{m, \ell+2 q+n+1}\left(\boldsymbol{x}_{m}\right)\left[L_{m, \ell+1}\left(\boldsymbol{x}_{m}\right)\right]^{2} \\
& -L_{m, \ell+q+n+1}\left(\boldsymbol{x}_{m}\right) L_{m, \ell+q+1}\left(\boldsymbol{x}_{m}\right) L_{m, \ell+1}\left(\boldsymbol{x}_{m}\right) \\
& -L_{m, \ell+n+1}\left(\boldsymbol{x}_{m}\right) L_{m, \ell+2 q+1}\left(\boldsymbol{x}_{m}\right) L_{m, \ell+1}\left(\boldsymbol{x}_{m}\right) \\
& +L_{m, \ell+n+1}\left(\boldsymbol{x}_{m}\right)\left[L_{m, \ell+q+1}\left(\boldsymbol{x}_{m}\right)\right]^{2},
\end{aligned}
$$




$$
\begin{aligned}
\mathcal{H}_{m, \ell, q, n}= & L_{m, \ell+2 q+n+1}\left(\boldsymbol{x}_{m}\right)\left[L_{m, \ell+1}\left(\boldsymbol{x}_{m}\right)\right]^{2} \\
& -2 L_{m, \ell+q+n+1}\left(\boldsymbol{x}_{m}\right) L_{m, \ell+q+1}\left(\boldsymbol{x}_{m}\right) L_{m, \ell+1}\left(\boldsymbol{x}_{m}\right) \\
& +L_{m, \ell+n+1}\left(\boldsymbol{x}_{m}\right)\left[L_{m, \ell+q+1}\left(\boldsymbol{x}_{m}\right)\right]^{2} \\
\mathcal{I}_{m, \ell, q, n}= & L_{m, \ell+2 q+n+1}\left(\boldsymbol{x}_{m}\right)\left[L_{m, \ell+1}\left(\boldsymbol{x}_{m}\right)\right]^{2} \\
& -2 L_{m, \ell+n+1}\left(\boldsymbol{x}_{m}\right) L_{m, \ell+2 q+1}\left(\boldsymbol{x}_{m}\right) L_{m, \ell+1}\left(\boldsymbol{x}_{m}\right) \\
& +L_{m, \ell+n+1}\left(\boldsymbol{x}_{m}\right)\left[L_{m, \ell+q+1}\left(\boldsymbol{x}_{m}\right)\right]^{2} .
\end{aligned}
$$

Then

$$
\begin{aligned}
& \mathcal{G}_{m, \ell, q, n} \geq 0, \quad \mathcal{H}_{m, \ell, q, n} \geq 0, \\
& \mathcal{H}_{m, \ell, q, n} \lesseqgtr \mathcal{G}_{m, \ell, q, n} \quad \text { when } q \lessgtr n, \\
& \mathcal{I}_{m, \ell, q, n} \geq \mathcal{G}_{m, \ell, q, n} \geq 0 \quad \text { when } n \geq q \text {. }
\end{aligned}
$$

Proof. In [27, Theorem 1 and Remark 2], it was obtained that, if $f(t)$ is completely monotonic on $(0, \infty)$ and

$$
\begin{aligned}
G_{q, n}(t)= & (-1)^{n}\left\{f^{(n+2 q)}(t) f^{2}(t)-f^{(n+q)}(t) f^{(q)}(t) f(t)\right. \\
& \left.-f^{(n)}(t) f^{(2 q)}(t) f(t)+f^{(n)}(t)\left[f^{(q)}(t)\right]^{2}\right\}, \\
H_{q, n}(t)= & (-1)^{n}\left\{f^{(n+2 q)}(t) f^{2}(t)-2 f^{(n+q)}(t) f^{(q)}(t) f(t)+f^{(n)}(t)\left[f^{(q)}(t)\right]^{2}\right\}, \\
I_{q, n}(t)= & (-1)^{n}\left\{f^{(n+2 q)}(t) f^{2}(t)-2 f^{(n)}(t) f^{(2 q)}(t) f(t)+f^{(n)}(t)\left[f^{(q)}(t)\right]^{2}\right\}
\end{aligned}
$$

for $n, q \in \mathbb{N}$, then

$$
\begin{gathered}
G_{q, n}(t) \geq 0, \quad H_{q, n}(t) \geq 0, \\
H_{q, n}(t) \lesseqgtr G_{q, n}(t) \quad \text { when } q \lessgtr n, \\
I_{q, n}(t) \geq G_{q, n}(t) \geq 0 \quad \text { when } n \geq q .
\end{gathered}
$$

Replacing $f(t)$ by $(-1)^{\ell}\left[H^{\prime}\left(t ; \boldsymbol{x}_{m}\right)\right]_{t}^{(\ell)}$ in $G_{q, n}(t), H_{q, n}(t)$, and $I_{q, n}(t)$ and simplifying produce

$$
\begin{aligned}
G_{q, n}(t)= & (-1)^{\ell+n}\left\{\left[H^{\prime}\left(t ; \boldsymbol{x}_{m}\right)\right]_{t}^{(\ell+2 q+n)}\left[\left[H^{\prime}\left(t ; \boldsymbol{x}_{m}\right)\right]_{t}^{(\ell)}\right]^{2}\right. \\
& -\left[H^{\prime}\left(t ; \boldsymbol{x}_{m}\right)\right]_{t}^{(\ell+q+n)}\left[H^{\prime}\left(t ; \boldsymbol{x}_{m}\right)\right]_{t}^{(\ell+q)}\left[H^{\prime}\left(t ; \boldsymbol{x}_{m}\right)\right]_{t}^{(\ell)} \\
& -\left[H^{\prime}\left(t ; \boldsymbol{x}_{m}\right)\right]_{t}^{(\ell+n)}\left[H^{\prime}\left(t ; \boldsymbol{x}_{m}\right)\right]_{t}^{(\ell+2 q)}\left[H^{\prime}\left(t ; \boldsymbol{x}_{m}\right)\right]_{t}^{(\ell)} \\
& \left.+\left[H^{\prime}\left(t ; \boldsymbol{x}_{m}\right)\right]_{t}^{(\ell+n)}\left[\left[H^{\prime}\left(t ; \boldsymbol{x}_{m}\right)\right]_{t}^{(\ell+q)}\right]^{2}\right\} \\
H_{q, n}(t)= & (-1)^{\ell+n}\left\{\left[H^{\prime}\left(t ; \boldsymbol{x}_{m}\right)\right]_{t}^{(\ell+2 q+n)}\left[\left[H^{\prime}\left(t ; \boldsymbol{x}_{m}\right)\right]_{t}^{(\ell)}\right]^{2}\right. \\
& -2\left[H^{\prime}\left(t ; \boldsymbol{x}_{m}\right)\right]_{t}^{(\ell+q+n)}\left[H^{\prime}\left(t ; \boldsymbol{x}_{m}\right)\right]_{t}^{(\ell+q)}\left[H^{\prime}\left(t ; \boldsymbol{x}_{m}\right)\right]_{t}^{(\ell)} \\
& \left.+\left[H^{\prime}\left(t ; \boldsymbol{x}_{m}\right)\right]_{t}^{(\ell+n)}\left[\left[H^{\prime}\left(t ; \boldsymbol{x}_{m}\right)\right]_{t}^{(\ell+q)}\right]^{2}\right\}, \\
I_{q, n}(t)= & (-1)^{\ell+n}\left\{\left[H^{\prime}\left(t ; \boldsymbol{x}_{m}\right)\right]_{t}^{(\ell+2 q+n)}\left[\left[H^{\prime}\left(t ; \boldsymbol{x}_{m}\right)\right]_{t}^{(\ell)}\right]^{2}\right. \\
& -2\left[H^{\prime}\left(t ; \boldsymbol{x}_{m}\right)\right]_{t}^{(\ell+n)}\left[H^{\prime}\left(t ; \boldsymbol{x}_{m}\right)\right]_{t}^{(\ell+2 q)}\left[H^{\prime}\left(t ; \boldsymbol{x}_{m}\right)\right]_{t}^{(\ell)} \\
& \left.+\left[H^{\prime}\left(t ; \boldsymbol{x}_{m}\right)\right]_{t}^{(\ell+n)}\left[\left[H^{\prime}\left(t ; \boldsymbol{x}_{m}\right)\right]_{t}^{(\ell+q)}\right]^{2}\right\} .
\end{aligned}
$$

Further taking $t \rightarrow 0^{+}$reveals

$$
\lim _{t \rightarrow 0^{+}} G_{q, n}(t)=\mathcal{G}_{m, \ell, q, n}, \quad \lim _{t \rightarrow 0^{+}} H_{q, n}(t)=\mathcal{H}_{m, \ell, q, n}, \quad \lim _{t \rightarrow 0^{+}} I_{q, n}(t)=\mathcal{I}_{m, \ell, q, n} .
$$


Substituting these quantities into 3.11 and simplifying bring about inequalities in 3.10 . The proof of Theorem 3.5 is complete.

Remark 3.1. When taking $x_{1}=\cdots=x_{m}=1$, all results in this section become conclusions for multi-order exponential numbers $L_{m, n}$.

\section{REFERENCES}

[1] N. Asai, I. Kubo, and H.-H. Kuo, Bell numbers, log-concavity, and log-convexity, Acta Appl. Math. 63 (2000), no. 1-3, 79-87; Available online at https://doi.org/10.1023/A: 1010738827855

[2] E. T. Bell, Exponential numbers, Amer. Math. Monthly 41 (1934), no. 7, 411-419; Available online at http://dx.doi.org/10.2307/2300300

[3] E. T. Bell, Exponential polynomials, Ann. of Math. (2) 35 (1934), no. 2, 258-277; Available online at http://dx.doi.org/10.2307/1968431

[4] K. N. Boyadzhiev, Exponential polynomials, Stirling numbers, and evaluation of some gamma integrals, Abstr. Appl. Anal. 2009, Art. ID 168672, 18 pp; Available online at http://dx. doi.org/10.1155/2009/168672.

[5] E. R. Canfield, Engel's inequality for Bell numbers, J. Combin. Theory Ser. A 72 (1995), no. 1, 184-187; Available online at https://doi.org/10.1016/0097-3165(95) 90033-0

[6] L. Comtet, Advanced Combinatorics: The Art of Finite and Infinite Expansions, Revised and Enlarged Edition, D. Reidel Publishing Co., Dordrecht and Boston, 1974.

[7] A. Dil and V. Kurt, Investigating geometric and exponential polynomials with Euler-Seidel matrices. J. Integer Seq. 14 (2011), no. 4, Article 11.4.6, 12 pp.

[8] B.-N. Guo and F. Qi, An explicit formula for Bell numbers in terms of Stirling numbers and hypergeometric functions, Glob. J. Math. Anal. 2 (2014), no. 4, 243-248; Available online at https://doi.org/10.14419/gjma.v2i4.3310

[9] W.-X. Ma, Bilinear equations, Bell polynomials and linear superposition principle, J. Phys. Conf. Ser. 411 (2013), no. 1, Aricle ID 012021, 11 pages; Available online at https://doi. org/10.1088/1742-6596/411/1/012021.

[10] W.-X. Ma, Bilinear equations and resonant solutions characterized by Bell polynomials, Rep. Math. Phys. 72 (2013), no. 1, 41-56; Available online at https://doi.org/10.1016/ S0034-4877(14)60003-3

[11] W.-X. Ma, Trilinear equations, Bell polynomials, and resonant solutions, Front. Math. China 8 (2013), no. 5, 1139-1156; Available online at https://doi.org/10.1007/ s11464-013-0319-5

[12] F. Mirzaee, Numerical solution of nonlinear Fredholm-Volterra integral equations via Bell polynomials, Comput. Methods Differ. Equ. 5 (2017), no. 2, 88-102.

[13] D. S. Mitrinović, Analytic Inequalities, Springer-Verlag, 1970.

[14] D. S. Mitrinović and J. E. Pečarić, On two-place completely monotonic functions, Anzeiger Öster. Akad. Wiss. Math.-Natturwiss. Kl. 126 (1989), 85-88.

[15] D. S. Mitrinović, J. E. Pečarić, and A. M. Fink, Classical and New Inequalities in Analysis, Kluwer Academic Publishers, 1993; Available online at https://doi.org/10.1007/ 978-94-017-1043-5

[16] J. E. Pečarić, Remarks on some inequalities of A. M. Fink, J. Math. Anal. Appl. 104 (1984), no. 2, 428-431; Available online at https://doi.org/10.1016/0022-247X(84) 90006-4

[17] F. Qi, An explicit formula for the Bell numbers in terms of the Lah and Stirling numbers, Mediterr. J. Math. 13 (2016), no. 5, 2795-2800; Available online at https://doi.org/10. 1007/s00009-015-0655-7.

[18] F. Qi, Some inequalities for the Bell numbers, Proc. Indian Acad. Sci. Math. Sci. 127 (2017), no. 4, 551-564; Available online at https://doi.org/10.1007/s12044-017-0355-2

[19] F. Qi, Some properties of the Touchard polynomials, ResearchGate Working Paper (2017), available online at https://doi.org/10.13140/RG.2.2.30022.16967

[20] F. Qi, D. Lim, and B.-N. Guo, Explicit formulas and identities for the Bell polynomials and a sequence of polynomials applied to differential equations, Rev. R. Acad. Cienc. Exactas Fís. Nat. Ser. A Mat. RACSAM (2018), in press; Available online at https://doi.org/10.1007/ s13398-017-0427-2 
[21] F. Qi, D.-W. Niu, and B.-N. Guo, Some identities for a sequence of unnamed polynomials connected with the Bell polynomials, Preprints 2017, 2017080044, 10 pages; Available online at https://doi.org/10.20944/preprints201708.0044.v1

[22] F. Qi, D.-W. Niu, D. Lim, and B.-N. Guo, A unified generalization of the Bell numbers and the Touchard polynomials and its properties, ResearchGate Working Paper (2017), available online at https://doi.org/10.13140/RG.2.2.36733.05603

[23] F. Qi, X.-T. Shi, and F.-F. Liu, Expansions of the exponential and the logarithm of power series and applications, Arab. J. Math. (Springer) 6 (2017), no. 2, 95-108; Available online at https://doi.org/10.1007/s40065-017-0166-4.

[24] J. Quaintance and H. W. Gould, Combinatorial Identities for Stirling Numbers. The unpublished notes of H. W. Gould. With a foreword by George E. Andrews. World Scientific Publishing Co. Pte. Ltd., Singapore, 2016.

[25] R. L. Schilling, R. Song, and Z. Vondraček, Bernstein Functions-Theory and Applications, 2nd ed., de Gruyter Studies in Mathematics 37, Walter de Gruyter, Berlin, Germany, 2012; Available online at https://doi.org/10.1515/9783110269338

[26] H. van Haeringen, Completely monotonic and related functions, J. Math. Anal. Appl. 204 (1996), no. 2, 389-408; Available online at https://doi.org/10.1006/jmaa.1996.0443

[27] H. van Haeringen, Inequalities for real powers of completely monotonic functions, J. Math. Anal. Appl. 210 (1997), no. 1, 102-113; Available online at https://doi.org/10.1006/jmaa. 1997.5376

[28] D. V. Widder, The Laplace Transform, Princeton University Press, Princeton, 1946.

$U R L:$ https: //qifeng618.wordpress.com 